because the growth of PI3K-p110a driven breast cancer allografts in mice, which was halted by the ketogenic diet and BYL-719, was rescued by the supplementation of exogenous insulin. Similar benefits in response to the ketogenic diet and PI3K inhibition using a range of inhibitors were observed in several patient-derived tumour xenograft models or syngeneic tumour allograft models of endometrial adenocarcinoma, bladder cancer and acute myeloid leukaemia (AML), in which PI3K-p110a signalling was driving growth. However, the therapeutic benefit of the ketogenic diet prior to PI3K blockade varied across models, and showed adverse effects when given alone in the AML model.

While insight into systemic glucose-insulin feedback will inform clinical practice, caution needs to be taken when translating these findings into a treatment strategy in the clinic.

Ulrike Harjes

ORIGINAL ARTICLE Hopkins, B. D. et al Suppression of insulin feedback enhances the efficacy of PI3K inhibitors. Nature https://doi.org/ 10.1038/541586-018-0343-4 (2018)

co-cultured with NS-F10 cells. Similar results were also observed in another pair of subclones isolated from a different DIPG tumour.

In vivo, bulk tumour cells and NS-F10 cells formed tumours that infiltrated the central nervous system more than tumours derived from NS-F8 cells, and mice with NS-F8-derived tumours survived longer. In addition, tumours grown from NS-F8 and NS-F10 co-cultured cells were more infiltrative than those made only of NS-F8 cells; importantly, neither cell population out-competed the other, suggesting that cooperation operates in vivo.

This work examined only two sets of subclones, but provides a rational explanation for why heterogeneity is maintained in DIPG and pGBM. As crucial subclones can be rare in the overall tumour, this study also has implications for tumour profiling studies and therapeutics.

Sarah Seton-Rogers

ORIGINAL ARTICLE Vinci, M. et al. Functiona diversity and cooperativity between subclonal populations of pediatric glioblastoma and diffuse intrinsic pontine glioma cells. Nat. Med. https:// doi.org/10.1038/s41591-018-0086-7 (2018)

\section{LUNG CANCER}

\section{Second chances}

Mutually exclusive activating mutations in KRAS and epidermal growth factor receptor (EGFR) are the most abundant driver mutations in lung adenocarcinomas, the most frequent histological subtype of non-small-cell lung cancer (NSCLC). Yet, mutant KRAS has so far proven to be an undruggable target and EGFR-selective inhibitors have shown little or no benefit to patients with KRAS-driven lung cancer. This has led to the misconception that mutations in KRAS confer independence from upstream regulation by ERBB family receptor tyrosine kinase (RTK) signalling. However, two groups have now independently established that RTK signalling is active in and necessary for KRAS-driven lung tumorigenesis.

To revisit the role of EGFR-related signalling in KRAS-driven lung cancers, both groups of researchers generated mouse models. Kruspig et al. used an inducible autochthonous mouse model of lung adenocarcinoma driven by endogenously expressed $K_{r a s}{ }^{\mathrm{G} 12 \mathrm{D}}$ combined with modest overexpression of Myc (KM mice). Analysis of tumour samples collected from these mice 6 weeks after induction showed increased mRNA expression of Erbb2, Erbb3 and multiple cognate ligands as well as phosphorylated ERBB proteins indicative of active signalling. Furthermore, comparison of these early tumours with KM tumours examined after 4-6 months revealed increased expression of phospho-ERK, associated with increased expression of ERBB ligands and RTK accessory proteins; a finding suggestive of amplified ERBB network signalling sustaining RAS pathway activity during KRAS-mutant lung cancer progression. In agreement, Moll et al. analysed publicly available datasets of human KRAS-mutated lung adenocarcinoma and adjacent normal lung tissue biopsy samples and found mRNA upregulation of ERBB RTKs and several of their ligands in the tumour tissue.

The conclusion that the activity of mutated KRAS relies on upstream signalling was also reached by Moll et al. by deleting Egfr in a mouse model of autochthonous lung cancer driven by $\operatorname{Kras}^{\mathrm{G} 12 \mathrm{D}}$ (KE mice). These mice had reduced tumour cell proliferation and decreased tumour ERK activation 10 weeks after tumour induction, and prolonged survival. Moreover, gene signature analysis of primary alveolar cells isolated from KE mice and induced in vitro demonstrated that deletion of Egfr impaired mutant KRAS activity.

To confirm that ERBB signalling is functionally required for lung tumour development, Kruspig et al. treated KM mice with the pan-ERBB inhibitor neratinib. This resulted in decreased activity of ERBB RTKs, reduced tumour cell proliferation and increased apoptosis, effects that were not

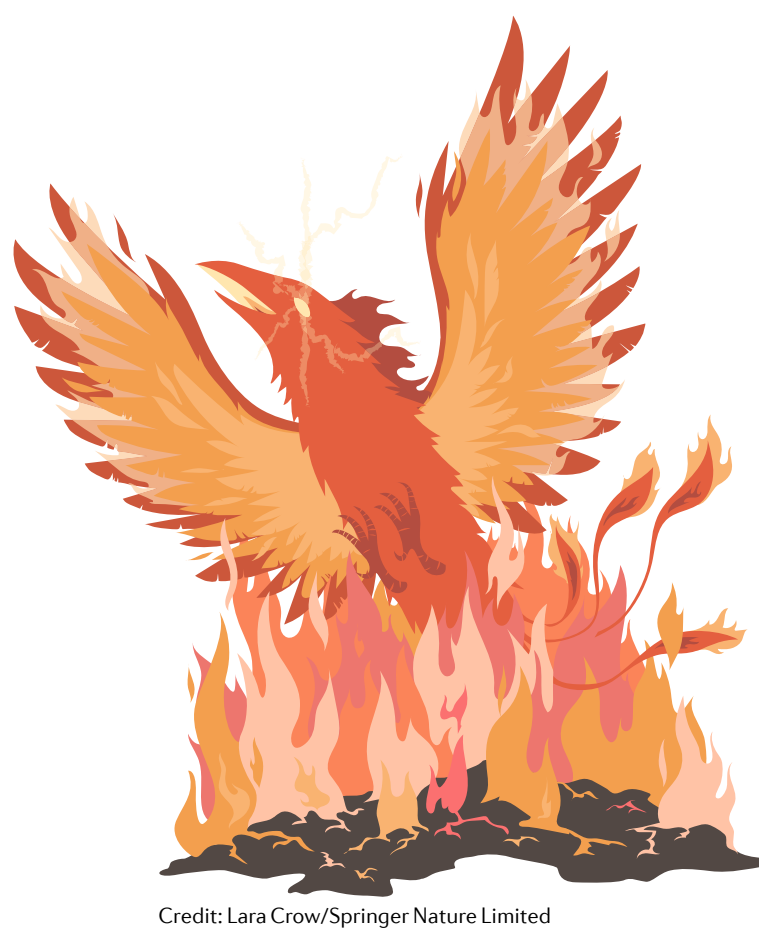

observed with the EGFR-selective inhibitor erlotinib. However, transient ERBB inhibition alone failed to improve the overall survival of KM mice but when used in combination with a MEK inhibitor trametinib could extend the survival beyond that attained with MEK blockade alone.

Similarly, Moll et al. found that treatment of mice with the pan-ERBB inhibitor afatinib could decrease the growth of tumours formed from human KRAS-mutated lung cancer A549 and A427 cell line-derived xenografts as well as a $K R A S^{\mathrm{G} 12 \mathrm{C}}$-mutated patient-derived xenograft. In addition, autochthonous lung tumours in a $\mathrm{Kras}^{\mathrm{G}}{ }^{12 \mathrm{D}}$-driven mouse model ( $\mathrm{K}$ mice) were also sensitive to afatinib but not erlotinib or gefitinib (another EGFR-selective inhibitor), resulting in reduced tumour burden and importantly decreased ERK activation. Since there was a compensatory mRNA upregulation of non-EGFR ERBB RTKs in tumours of KE mice 20 weeks after induction leading to a recovery of the tumour proliferation rate, Moll et al. tested the effects of afatinib on tumours generated from EGFR-deficient A549 xenografts in mice. This treatment was sufficient to block tumour growth, confirming that afatinib could overcome the tumour escape mechanism mediated by ERBB RTKs.

Together these two studies present a strong case for re-evaluating the use of the US Food and Drug Administration (FDA)-approved pan-ERBB inhibitors in clinical trials to treat patients with KRAS-driven NSCLC.

Anna Dart

ORIGINAL ARTICLES Kruspig, B. et al. The ERBB network facilitates KRAS-driven lung tumorigenesis. Sci. Transl Med. 10, eaao2565 (2018) | Moll, H. P. et al. Afatinib restrains K-RAS-driven lung tumorigenesis. Sci. Transl Med. 10, eaao2301 (2018) 